\title{
Controlled electromigration protocol revised
}

Vyacheslav S. Zharinov, Xavier D. A. Baumans, Alejandro V. Silhanek, Ewald Janssens, and Joris Van de Vondel

Citation: Review of Scientific Instruments 89, 043904 (2018); doi: 10.1063/1.5011953

View online: https://doi.org/10.1063/1.5011953

View Table of Contents: http://aip.scitation.org/toc/rsi/89/4

Published by the American Institute of Physics

\section{Articles you may be interested in}

Electromigration and the structure of metallic nanocontacts

Applied Physics Reviews 4, 031302 (2017); 10.1063/1.4994691

Quantitative magneto-optical investigation of superconductor/ferromagnet hybrid structures

Review of Scientific Instruments 89, 023705 (2018); 10.1063/1.5016293

In situ/non-contact superfluid density measurement apparatus

Review of Scientific Instruments 89, 043901 (2018); 10.1063/1.5012043

Improving the viability and versatility of the $\mathrm{E} \times \mathrm{B}$ probe with an active cooling system

Review of Scientific Instruments 89, 043502 (2018); 10.1063/1.5004469

Analysis of retarding field energy analyzer transmission by simulation of ion trajectories

Review of Scientific Instruments 89, 043501 (2018); 10.1063/1.5018269

Note: Commercial SQUID magnetometer-compatible NMR probe and its application for studying a quantum magnet

Review of Scientific Instruments 89, 046101 (2018); 10.1063/1.5023675

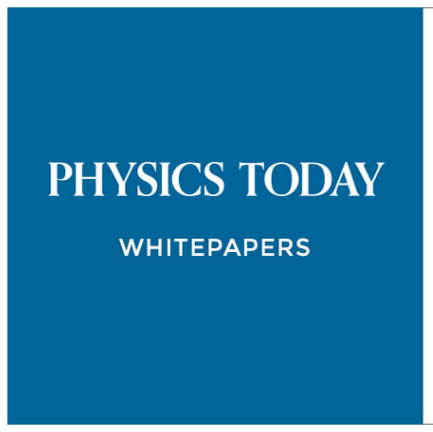

MANAGER'S GUIDE

Accelerate R\&D with

Multiphysics Simulation
READ NOW

PRESENTED BY

$\checkmark \subset \bigcirc M S O L$ 


\title{
Controlled electromigration protocol revised
}

\author{
Vyacheslav S. Zharinov, ${ }^{1}$ Xavier D. A. Baumans, ${ }^{2}$ Alejandro V. Silhanek, ${ }^{2}$ Ewald Janssens, ${ }^{1}$ \\ and Joris Van de Vondel ${ }^{1, a)}$ \\ ${ }^{1}$ Laboratory of Solid State Physics and Magnetism, Department of Physics and Astronomy, KU Leuven, \\ Celestijnenlaan 200D, B-3001 Leuven, Belgium \\ ${ }^{2}$ Experimental Physics of Nanostructured Materials, Q-MAT, CESAM, Université de Liège, B-4000 Sart Tilman, \\ Belgium
}

(Received 3 November 2017; accepted 15 March 2018; published online 11 April 2018)

\begin{abstract}
Electromigration has evolved from an important cause of failure in electronic devices to an appealing method, capable of modifying the material properties and geometry of nanodevices. Although this technique has been successfully used by researchers to investigate low dimensional systems and nanoscale objects, its low controllability remains a serious limitation. This is in part due to the inherent stochastic nature of the process, but also due to the inappropriate identification of the relevant control parameters. In this study, we identify a suitable process variable and propose a novel control algorithm that enhances the controllability and, at the same time, minimizes the intervention of an operator. As a consequence, the algorithm facilitates the application of electromigration to systems that require exceptional control of, for example, the width of a narrow junction. It is demonstrated that the electromigration rate can be stabilized on pre-set values, which eventually defines the final geometry of the electromigrated structures. Published by AIP Publishing. https://doi.org/10.1063/1.5011953
\end{abstract}

\section{INTRODUCTION}

The investigation of the electromigration (EM) process started in the 1960s as a result of its destructive impact on the wiring of integrated circuits. ${ }^{1,2}$ The origin of this phenomenon is the transfer of material that is subjected to high current densities due to the so-called "wind" force of moving charge carriers and the applied electrostatic force. This process is accompanied by stress-induced migration and, to a less extent, by thermomigration. ${ }^{3,4}$ Besides its obvious drawbacks, the EM process has found several applications in nanoscience and nanotechnology. Since it is possible to constrain EM spatially, it can be used for local, in situ, nanosculpting. ${ }^{5}$ This allows one to fabricate devices with reduced dimensionality that exhibit quantum transport properties ${ }^{6}$ or tunneling phenomena in nanogaps. ${ }^{7}$ Thanks to these merits, EM has been used, for instance, in charge transport studies of single molecules. ${ }^{8}$ In contrast to extensively used optical and electron beam lithography, EM-based fabrication does not need resist polymer coatings. This excludes possible contamination of fabricated junctions by chemical residuals. Moreover, EM can be performed under extreme conditions like cryogenic temperatures and high vacuum, ${ }^{9}$ which in turn allows us to drastically reduce the contamination and/or oxidation of the metallic structure.

A considerable drawback of EM-based nanofabrication is the poor controllability of the process. Substantial efforts have been made in order to improve the reliability of EM by adding an analogous proportional-integral-derivative (PID) feedback loop into the EM controlling scheme ${ }^{10,11}$ or by drastically increasing the reaction speed of the control system. ${ }^{12}$ Here we demonstrate that an improved performance

a) Author to whom correspondence should be addressed: joris.vandevondel@ kuleuven.be of EM-based fabrication can be achieved by a successful revision of the EM protocol, instead of hardware redesign. An algorithm is proposed, which is particularly sensitive when the internal positive feedback of the EM process is about to break the junction. In addition, the algorithm is able to maintain EM at a reasonably low and stable rate. Using this capability, we reveal the impact of the EM speed on the resulting geometry of the metallic constriction. At low EM rates, mass migration is triggered in the vicinity of the narrowest point resulting in a reduction of its width. At higher EM rates, a very different behavior is observed. In this case, electromigration is triggered far away from the constriction region and the migrated atoms are collected at the constriction. The observed difference directly shows the subtle dependence of the EM process on the exact out-of-equilibrium conditions that can be set by the proposed protocol.

\section{CONCEPTUAL DESCRIPTION OF ELECTROMIGRATION}

In order to get a general understanding of the EM process, we use the simplified description that was first introduced by Black. ${ }^{13}$ In Black's model, the mass transport flux, $\Phi$, which is the amount of material removed per unit of cross section and unit of time, is given by

$$
\Phi=A j^{2} \exp \left(-\frac{E_{A}}{k_{B T}}\right),
$$

where $E_{A}$ is the activation energy or barrier needed to overcome by an atom in the material to start diffusing, $T$ is the local temperature, $k_{B}$ is the Boltzmann constant, $j$ is the current density, and $A$ is a material and process dependent constant. The $j^{2}$ term is the result of the momentum transfer from the conduction electrons to the thermally activated ions. The activated ion 
density, defined by the exponent, follows an Arrhenius behavior. This indicates that the Joule heating, resulting in a local temperature increase, has a decisive role in the electromigration process. ${ }^{14}$ Tailoring a region with a reduced cross section, i.e., the constriction, via e-beam lithography, one can locally increase the current density and, consequently, the temperature. Therefore, EM will be triggered nearby the narrowest region of the transport bridge.

Moreover, in case EM starts at the constriction, its cross section will reduce and both the current density and dissipated power (proportional to the local temperature) will increase (in the case of $I=$ const ). Both contributions result into a positive feedback to the EM process. The exponential dependence of the EM speed on temperature can have a destructive impact in case the process is not actively damped. Therefore, using EM as a controlled fabrication method requires a well-thought combination of monitoring and steering of the process. The aim of our software is to prevent the runaway of the EM process and keep the outgoing mass transport flux $\Phi$, directly associated with the speed of electromigration, nearly constant throughout the process.

In the literature, several process variables have been considered to control and monitor the electromigration process in different devices, such as $d r / d t,{ }^{15,16}(d r / d t) / r,{ }^{11,17} d(1 / r) / d t,{ }^{6,18}$ or $P_{r},{ }^{19}$ with $r$ and $P_{r}$ as the resistance of and the dissipated power in the device, respectively. Although all these variables are related to the speed of electromigration, a solid motivation for the particular selected process variable is lacking in most references. In order to properly identify the suitable process variable, which permits to gain control over the EM process, it is important to first understand the consequences of EM on the properties of the system.

In the particular case of $\mathrm{Al}$ and $\mathrm{Au}$ constrictions, discussed in this work, scanning electron microscopy images reveal a progressive reduction of the cross section during the EM process. The consequent change of geometry affects a region, which we will call a junction, of length $l$ and resistivity $\rho$. Both the parameters are assumed constant throughout the process, while the cross section $\sigma$ can vary. The electromigration of this region is the result of an outgoing mass transport flux. Under the aforementioned assumptions, $\Phi$ is proportional to the change of the cross-sectional area $\sigma$ divided by the total cross section, $\Phi \propto(d \sigma / d t) / \sigma=\dot{\sigma} / \sigma$ (using shorthand dot notation for the time derivative). Since the conductance of the junction is $G_{J}=\sigma / \rho l$, we obtain $\Phi(t) \propto-\dot{G}_{J} / G_{J}=\dot{R}_{J} / R_{J}$. Based on these considerations, we consider $-\dot{G}_{J} / G_{J}$ as a measure for the EM speed and adopt it as the process variable to monitor and control the time evolution of EM.

\section{REALIZATION OF A CONSTANT EM SPEED}

In this section, we implement the above-described concepts and demonstrate the ability of our protocol to electromigrate at a constant speed. Figure 1(a) shows an SEM image of a nanoconstriction together with the electrical scheme. Here $U_{0}$ is the voltage bias applied to the whole system, $I$ is the current through the nanoconstriction, $R_{L}$ is the lead resistance, $U$ is the voltage measured using a four probe geometry in order to

(a)
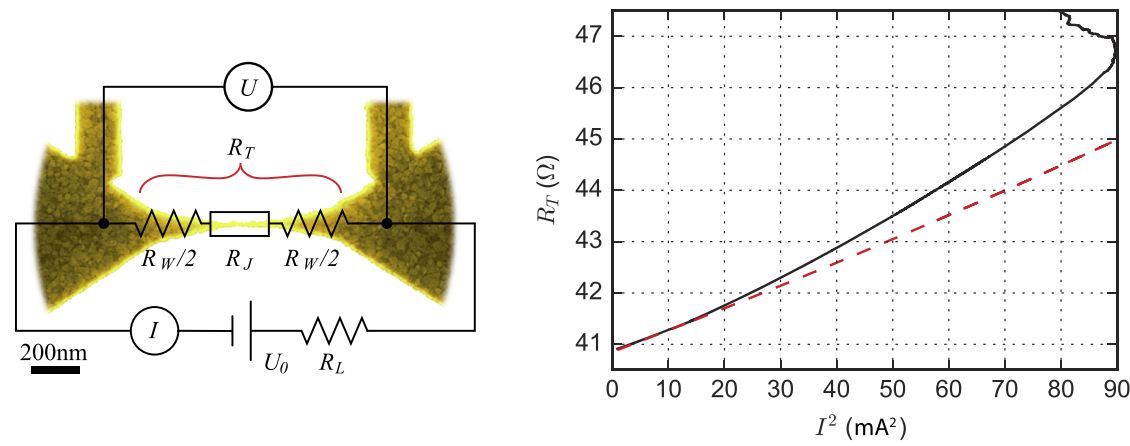

(b)
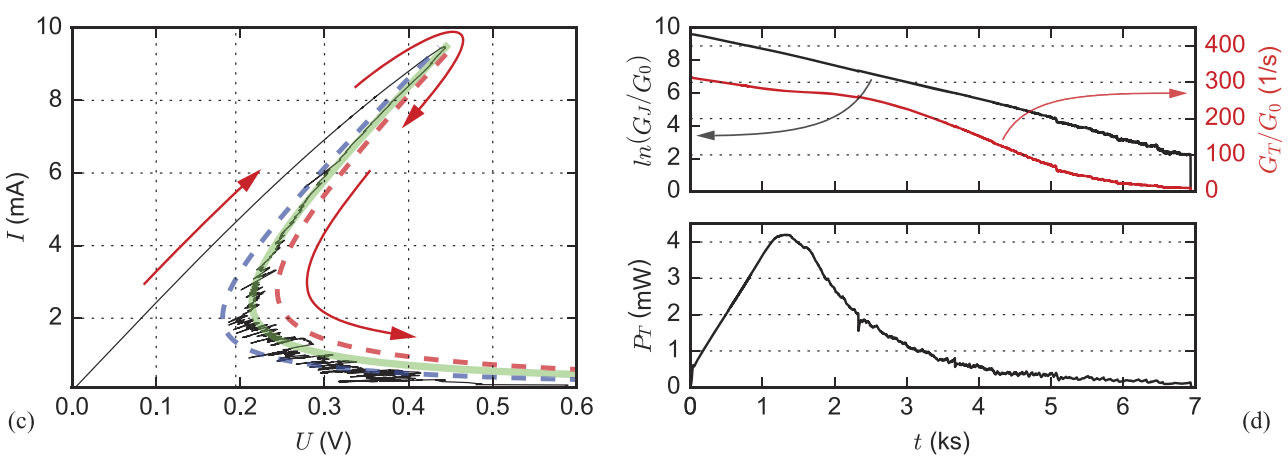

FIG. 1. (a) Electrical scheme of a representative gold nanodevice used for EM. Background: the SEM image of a sample junction prepared for EM. (b) Total resistance $R_{T}=R_{W}+R_{J}$ as a function of $I^{2}$ during the EM process (black line). The red dashed line is the extrapolated curve fitted in the range 0-14 $\mathrm{mA}^{2}$, where the sweep is assumed to be fully reversible with the linear relation between $R_{T}$, the temperature of the device, and the total dissipation power. (c) The black line is an experimentally obtained $I(U)$ curve during EM for a rate $-\dot{G}_{J} / G_{J}=0.001 \mathrm{~Hz}$. The green curve is a fit of the data using the constant power model $\left(P_{J}^{*}=0.26 \mathrm{~mW}\right.$ and $R_{W}^{*}=44 \Omega$ ). Red and blue dashed lines schematically correspond to the maximal and minimal EM speeds, at which the process can be controlled. Red arrows indicate the chronological evolution of the EM progress. (d) Black and red lines in the top graph represent $\ln \left(G_{J} / G_{0}\right)$ and $d G / d t$ as a function of time, respectively. The bottom graph represents the total power dissipated as a function of time during the EM process. 
eliminate the impact of the leads, $R_{J}$ is the junction resistance (resistance of the electromigrated region), and $R_{W}$ is the wire resistance between voltage probes excluding the junction.

In order to obtain the suggested process variable, $-\dot{G}_{J} / G_{J}=\dot{R}_{J} / R_{J}$, one requires $R_{J}$, while only $R_{T}=R_{J}+$ $R_{W}=U / I$ is directly obtained via a four point measurement [Fig. 1(a)]. In order to separate $R_{J}$ and $R_{W}$ contributions to the total resistance of the device, it is necessary to estimate $R_{J}$ before EM and use that value for calculating $R_{W}$, which with the earlier given definition for the junction is unaffected by the EM mass transport. This can be done by carefully analyzing scanning electron microscopy pictures of electromigrated devices in order to determine the electromigrated region and combine this information with the experimentally determined resistivity of the material for thin films. ${ }^{20}$ By doing so, we obtained $R_{J} \approx 1 \Omega$ at room temperature for the tested gold devices with a junction cross section near $200 \mathrm{~nm}^{2}$. Since the EM process is never perfectly the same, this estimate suffers from substantial uncertainty. The total resistance of the device $\left(R_{T}=41 \Omega\right)$, usually taken instead of $R_{J}$, would lead to an underestimation of the process variable $\left(-\dot{G}_{J} / G_{J}\right)$ up to three orders of magnitude in the device presented in Fig. 1(a).

Moreover, the calculation of the rate $-\dot{G}_{J} / G_{J}$ becomes more complicated when the Joule heating starts to affect $R_{T}$ at higher applied currents. In this case, the observed increase of $R_{T}$ could be incorrectly attributed to electromigration. Therefore, it is needed to distinguish temperature-induced changes of $R_{T}$ from changes induced by EM of $R_{J}$. Analyzing Fourier's heat transfer law and solving it over the device, one can see that the total power dissipated, $P_{T}$, is proportional to the induced change of the device average temperature, $\Delta T .^{21,22}$ Assuming no EM takes place, the nearly linear temperature dependence of the resistivity results in an almost linear increase of $R_{T}$ as a function of the total power $P_{T}=I^{2} R_{T}(I)$,

$$
R_{T}(I)=R_{T 0}\left(1+\alpha I^{2} R_{T}(I)\right) .
$$

This is indeed confirmed experimentally by the dependence of $R_{T}$ on $I^{2}$ for a fast current sweep until $4 \mathrm{~mA}$ that can be fitted and extrapolated [red dashed line in Fig. 1(b)] as given by

$$
R_{T}(I)=\frac{R_{T 0}}{\left(1-R_{T 0} \alpha I^{2}\right)},
$$

where $R_{T 0}=41 \Omega$ is the total resistance at room temperature (zero current) and $\alpha=25 \Omega^{-1} \mathrm{~A}^{-2}$ for this particular device. Since $R_{W} \approx R_{T}$, we assume $R_{W}(I)$ to follow Eq. (3) with the same $\alpha$. The function $R_{W}(I)$ can be used for a more accurate estimation of $R_{J}$ throughout the process so that heating effects are compensated and, therefore, $-\dot{G}_{J} / G_{J}$ is associated mostly with the mass transport only. Once these numbers are obtained, we can calculate the process variable and start the EM process.

Figure 1(c) shows a typical $I(U)$ characteristics obtained during the electromigration process with a rate $-\dot{G}_{J} / G_{J}=$ $0.001 \mathrm{~Hz}$. Notice that $-\dot{G}_{J} / G_{J}=\frac{d}{d t}\left(\ln \left(G_{J} / G_{0}\right)\right)$ (with $G_{0}$ as the quantum of conductance); therefore, it is convenient to track the evolution of $\ln \left(G_{J} / G_{0}\right)$ in time. The black curve in Fig. 1(d) (top panel) shows $\ln \left(G_{J} / G_{0}\right)$ for the same process as in Fig. 1(c), and the bottom panel shows the total power dissipated through the electromigration process. The almost linear decrease of $\ln \left(G_{J} / G_{0}\right)$ indicates that the software is able to control the process variable at the desired value throughout the EM process.

The electromigration process can be divided into two different regions, depending on the time evolution of the current. In the first region (increasing $I$, until $9.5 \mathrm{~mA}$ ), the control algorithm increases the voltage in order to attain the requested $-\dot{G}_{J} / G_{J}$ setpoint. In this region $(t<1.3 \mathrm{ks})$, a linear increase of $P_{T}$ over time is observed [see Fig. 1(d), bottom panel]. Electromigration, which depends exponentially on temperature [see Eq. (1)], is not the main cause of the resistance change until reaching the point of the maximum current. Rather the strong increase of the local temperature, and the related reversible increase of $R_{J}$, plays an important role in achieving the setpoint. In addition, irreversible sample modifications also contribute to the increased resistance of the device. This can be verified by comparing the $R_{T}\left(I^{2}\right)$ curve for the extrapolated fast sweep [106 $\mu \mathrm{A} / \mathrm{s}$ between $0-14 \mathrm{~mA}^{2}$, dashed red line in Fig. 1(b), used to characterize the thermal properties of the device] and the slower sweep preceding electromigration [5 $\mu \mathrm{A} / \mathrm{s}$, black line in Fig. 1(b)]. The observed divergence between both curves can be partially explained by the presence of EM even before the maximum current is reached. These irreversible modifications should occur at the junction region, where both current density and temperature are maximal. By comparing the experimentally obtained $R_{T}$ value at maximum applied current with the one expected from the heating model [see Fig. 1(b)], a maximal increase of $1.5 \Omega$ is attributed to the junction resistance.

The second region (decreasing $I$ ) starts after a maximal current of $9.50 \mathrm{~mA}$ is reached. In this region, the electromigration is accelerated considerably. The crossover between the two regions is clearly identified by the changing $I(U)$ dependence [Fig. 1(c)], accompanied by reduction of the total dissipated power, $P_{T}$ [Fig. 1(d), bottom panel]. Once the electromigration starts, the temperature of the junction is expected to remain nearly constant (evidence is given below). However, the power dissipated in the wires drastically reduces with EM, which implies that the wires cool down. The effect of the associated reduction of $R_{W}(I)$ is taken into account by applying Eq. (3).

In order to confirm the constant temperature of the junction area within the second EM region, the measured $I(U)$ data are fitted using a model assuming a constant power dissipated at the junction. ${ }^{6,17}$ This model considers the structure as two resistors connected in series as already illustrated in Fig. 1(a). Using the notation introduced before $(*$ indicates that the values were obtained using the constant power model),

$$
U=I R_{W}^{*}+P_{J}^{*} / I \text { with } P_{J}^{*}, R_{W}^{*}=\text { const, }
$$

where $P_{J}^{*}$ is the power dissipated in the junction area, $R_{T}=R_{W}^{*}+R_{J}^{*}$. The obtained fit is presented in Fig. 1(c) by the green line. The model curve reproduces satisfactorily the experiment with $P_{J}^{*}=0.26 \mathrm{~mW}$ and $R_{W}^{*}=44 \Omega$, indicating that it captures the main features of the EM. This model assumes that the dissipated power in the junction region, $P_{J}^{*}$, remains constant during electromigration, ${ }^{6,17}$ while the power 
dissipated in the wires, $P_{W}^{*}$, has no impact on the EM. This was explained by the fact that EM is ruled by the temperature of the junction, which was assumed to be proportional to the heat dissipated in the junction region. ${ }^{6,18}$ While narrowing the junction, the EM process also reduces its heat conductivity, which is known to be reciprocal to the junction temperature increase. Therefore, less power will be needed to maintain the same temperature. This behavior was confirmed in Ref. 14 by the direct measurement of the junction temperature at different stages of the electromigration. As such, the fitting works very well, although one has to be careful with quantitative interpretation of the obtained fitting parameters. Nevertheless, $R_{J}^{*}$ calculated from the fitting parameters for the transition point $(I=9.5 \mathrm{~mA})$ between two EM regions gives $2.9 \Omega$, which is found to be close to $R_{J}(I=9.5 \mathrm{~mA})=R_{T}-R_{W}$ $(I=9.5 \mathrm{~mA})=R_{J}\left(T_{\text {Room }}\right)+\Delta R_{J}(I=9.5 \mathrm{~mA}) \approx 1 \Omega$ $+1.5 \Omega=2.5 \Omega$.

To summarize, the junction temperature can be controlled indirectly by the current flowing through the junction so that the Joule heating and heat sink compensate each other reaching dynamic equilibrium. This is achieved by using a modified feedback control system, which will be discussed in Sec. V. In addition, by using $-\dot{G}_{J} / G_{J}$ as a process variable, a welldefined speed of electromigration during the whole process can be maintained. However, the selected speed of electromigration is restricted to specific $P_{J}^{*}$ ranges, which are schematically given by the dashed lines in Fig. 1(c). The rightmost red $P_{J}^{*}$ curve corresponds to EM with too high rate, i.e., the feedback system is too slow to prevent thermal runaway and the sample can easily be blown up. The blue $P_{J}^{*}$ curve corresponds to EM with too slow rate, which is not practically usable and/or the changes in the process variable will not exceed the electronic noise level making speed setting unreliable.

\section{EXTENDING ELECTROMIGRATION TO OTHER PROCESS VARIABLES}

Inspired by the vast amount of process variables used in the literature, we tested and confirmed that the proposed software has the ability to electromigrate different devices using a broad range of process variables, including $-\dot{G}_{T}$, that implies a crude assumption of the whole device as a junction. Although a solid justification of the latter process variable cannot be made, it is much simpler to handle since no prior knowledge of the exact shape of the junction and its contribution to the total resistance is needed. To check the validity of the latter process variable, the evolution of $\ln \left(G_{J} / G_{0}\right)$ [Fig. 1(d); black line] and $G_{T} / G_{0}$ (red line) have been compared for the same electromigration run as shown in Fig. 1(c). Although both quantities do not have the same time dependence and they are not linked by a linear relation, a one to one correspondence exists between both process variables, which explains why $-\dot{G}_{T}$ could be successfully used to roughly control the EM speed. As a proof of principle, we electromigrated a similar sample using $-\dot{G}_{T}$ as a process variable. The corresponding $I(U)$ curve, provided in Fig. 2, is indeed very similar to the one obtained in Fig. 1(c) and can, as well, be properly fitted by the constant power model.

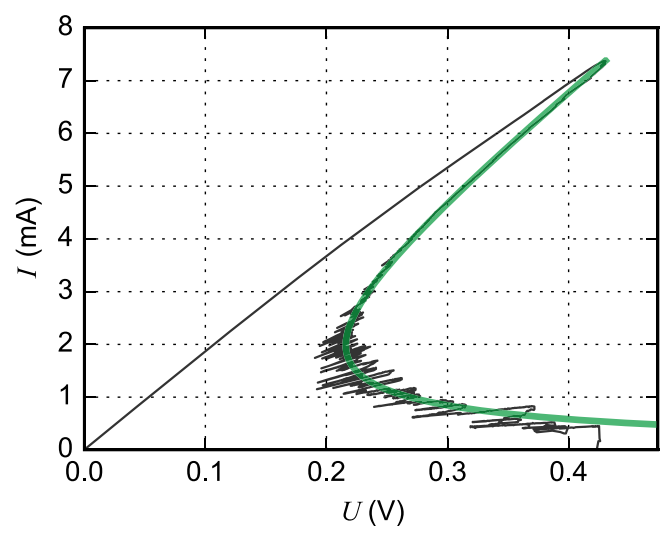

FIG. 2. $I(U)$ curve obtained using the process variable $\dot{G}_{T}(3.9 \mu \mathrm{S} / \mathrm{s}$, black line). The green line is a fit of $I(U)$ by the constant power model provided in Eq. (4), $P_{J}^{*}=0.21 \mathrm{~mW}$ and $R_{W}^{*}=54.4 \Omega$.

\section{CONTROL ALGORITHM}

In this section, the most important aspects of the control algorithm and its unique features compared to existing EM software are discussed. Although its implementation is rather straightforward, the algorithm allows us to electromigrate different devices toward a specific conductance value at a constant EM speed with minimal intervention by an operator. The general idea of most electromigration procedures in the literature is to increase the applied voltage until a threshold value of the selected process variable is obtained. Once this value is reached, the applied voltage is strongly reduced to prevent uncontrolled EM due to a positive feedback. ${ }^{6,23}$ This sequence is repeated until the desired nanoconstriction or nanogap is realized. The obvious advantages of this algorithm are simplicity and minimal needs for computer calculation power. Nevertheless, this procedure has several drawbacks. First, it lacks control over the process since the dissipated power, which defines the speed of electromigration, may vary strongly. This low level of speed control implies an irreproducible EM process since, as will be shown in Sec. VI, the final geometry of a junction depends on the EM rate. Second, the downtime (time during which no substantial EM is happening) is long. Finally, it is required to gradually increase the integration time of the process variable evaluation, since resistance fluctuations become higher with EM [see data in Fig. 1(c)]. This reduces the reaction speed, which is essential to deal with sudden resistance jumps. As a result, commonly used EM procedures demand the continuous attention of an operator correcting parameters during the whole EM process. Nevertheless, large fluctuations in the speed of electromigration will still be present.

In order to evade those limitations, we propose to replace the aforementioned feedback algorithm by a combination of a conventional software-based PID controller, defining the average change of the process variable, with a non-linear algorithm that reacts to sudden jumps in the process variable. The flow chart of the proposed algorithm is given in Fig. 3. The right loop (blue) is based on a conventional PID cycle. In notions of PID controller theory, the applied voltage bias is the control variable and the process variable (denoted by $p$ ) 


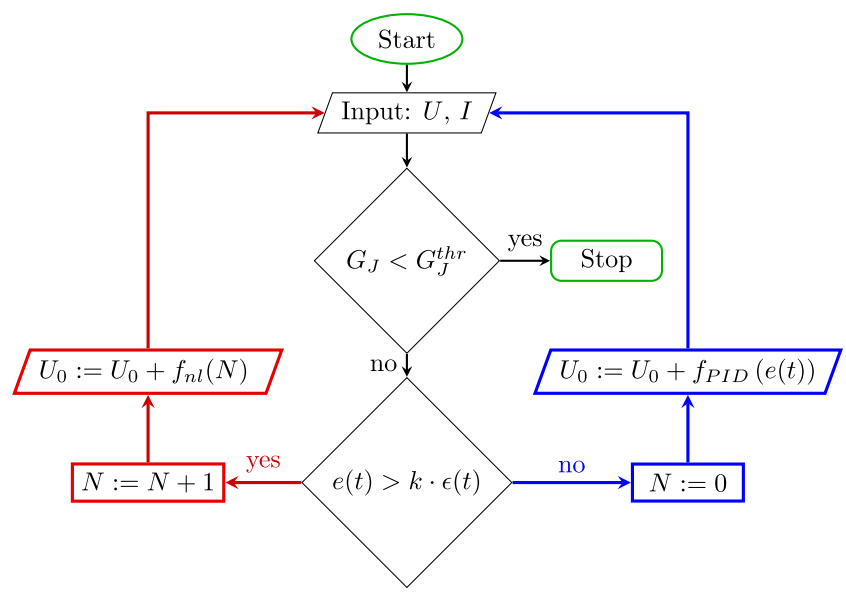

FIG. 3. Flow chart of the algorithm to control the EM process with control variable $U$ and process variable $p$. The applied bias $U_{0}$ is adjusted to keep $p$ as close as possible to the setpoint value $p_{\text {setp }}$ minimizing the error $e(t)=p-p_{\text {setp }}$. $f_{P I D}$ is a response function of the PID controller, whereas $f_{n l}$ is a non-linear algorithm response. $\epsilon(t)$ is the RMS noise value, $k$ is a numerical constant, and $N$ is a counter variable. The algorithm terminates if the conductance of the junction, $G_{J}$, reaches the pre-set threshold value $G_{J}^{t h r}$.

should be directly related to the electromigration speed, such as $-\dot{G}_{J} / G_{J}$ or $-\dot{G}_{T} \cdot{ }^{24}$ As in a conventional PID controller, the error between the setpoint and the measured process variable, $e(t)=p-p_{\text {setp }}$, is continuously monitored. The PID algorithm is based on proportional, integral, and derivative terms of $e(t)$ that are incorporated in the function $f_{P I D}(e(t))$ with corresponding coefficients, which should be adjusted for different devices, materials, and environmental conditions.

As discussed in Sec. III, the EM process is critically dependent on the temperature of the constriction, which is directly related to the dissipated power. A sudden change of resistance could result in an increased power dissipation and destruction of the junction. To deal with this positive feedback, it is important to react promptly to sudden changes of the sample resistance. PID controllers inherently do not work well with non-linear system response and are too slow to efficiently deal with this. To resolve this issue, a non-linear exponential response scheme was added, which is presented by the left loop (red) in the flow chart in Fig. 3. For every control cycle during which the condition $e(t)>k \cdot \epsilon(t)$ is satisfied, the reaction amplitude $\left(f_{n l}\right)$ is increased until the increase in the process variable is neutralized. Here, $\epsilon(t)$ is the noise RMS, with $k$ as a numerical constant. The threshold should be defined such that the algorithm does not react on noise. The reaction amplitude has the following form: $f_{n l}(N)=-\delta U_{0 \downarrow} \cdot a^{N}$, where $\delta U_{0 \downarrow}$ and $a$ are constants, while $N$ is the loop index counter. The exponential dependence on $N$ is added to be able to stop positive feedback of the EM process and to reduce the reaction amplitude on insignificant threshold crossings.

Figure 1(d) is a direct proof of the ability of the software to attain the setpoint, $p_{\text {setp }}$, throughout the electromigration process. The control cycles terminate once a certain threshold value of the conductance, $G_{J}^{t h r}$, is achieved. $G_{J}^{t h r}$ is directly related to the final cross section of the junction. Due to the high degree of control, the user can accurately estimate the

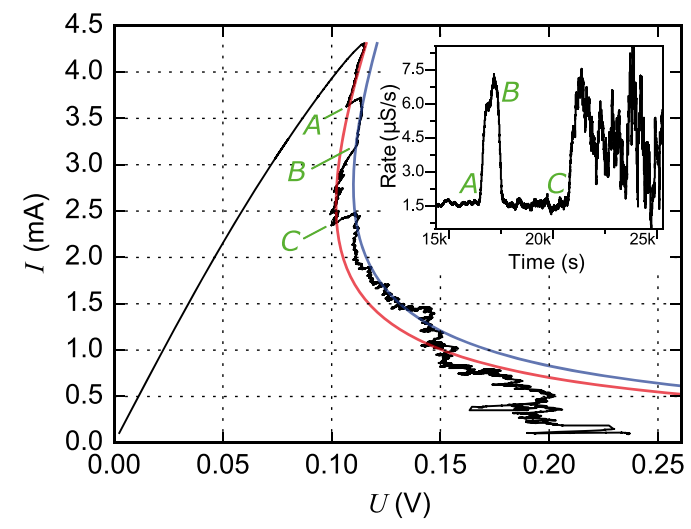

FIG. 4. The $I(U)$ curve during electromigration was fitted by the constant power model and provides $P_{J}^{*}$ values of $131 \mu \mathrm{W}$ (red curve for $\dot{G}_{T}=1.5 \mu \mathrm{S} / \mathrm{s}$ ) and $152 \mu \mathrm{W}$ (blue curve for $\dot{G}_{T}=6 \mu \mathrm{S} / \mathrm{s}$ ), respectively. The inset shows the time dependence of $\dot{G}_{T}$, demonstrating the possibility of the control algorithm to keep the process variable at the pre-set rates. Letters $A, B, C$ point to the moments of EM rate changes.

time needed to reach this value. The developed protocol has proved to be useful to study the evolution of superconductivity in nanojunctions of several different materials ${ }^{9,25,26,30}$ and electrically driven optical nanoantennas. ${ }^{27}$ In these cases, the EM software allowed us to change the conductance of a junction reproducibly and with high accuracy to attain the quantum conductance regime. For example, as shown in the inset of Fig. 2(h) of Ref. 25, we could bring the resistance within a targeted region of $0.25 \Omega$ during 12 consecutive runs of electromigration and anti-electromigration.

In order to demonstrate that the speed of electromigration can indeed be controlled and switched to different values during the same EM process, we electromigrated a device using $-\dot{G}_{T}$ as a process variable (shown in Fig. 4). During the electromigration process, the setpoint was changed from $-1.5 \mu \mathrm{S} / \mathrm{s}$ to $-6 \mu \mathrm{S} / \mathrm{s}$ and back, corresponding to constant dissipated powers of $131 \mu \mathrm{W}$ and $152 \mu \mathrm{W}$, respectively, as evaluated from constant power model fitting. Reproducible switching between the two different EM rates confirms the reliability of the proposed protocol.

\section{INFLUENCE OF THE EM SPEED ON THE GEOMETRY OF THE PROCESSED STRUCTURES}

The EM process depends on a subtle balance of counteracting forces and, therefore, it is expected that the speed of electromigration, related to the current density, temperature, and temperature gradient of the device during EM has a serious impact on the process. For instance, unstable EM may lead to nanoparticle appearance in the formed gap, ${ }^{17,29}$ which could cause the presence of parasite Kondo effect. ${ }^{17}$ To the best of our knowledge, this critical dependence has, so far, not been explored in detail. The proposed algorithm is particularly useful to tackle this issue. In Fig. 5, we present a striking demonstration of the influence of the EM rate on aluminum structures of the same initial design and the same external parameters for rates that differ almost one order of magnitude from each other. As can be seen in Fig. 5, the 


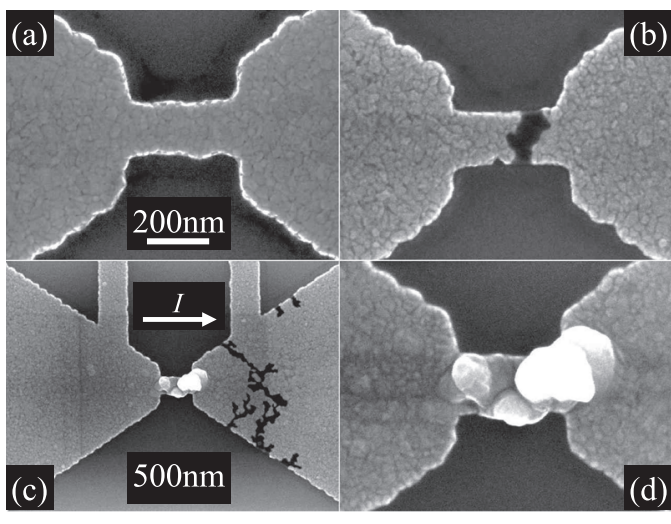

FIG. 5. SEM micrographs of the virgin junction (a) and after electromigration at low $\dot{G}_{T}=8 \mu \mathrm{S} / \mathrm{s}$ (b) and high $\dot{G}_{T}=60 \mu \mathrm{S} / \mathrm{s}[(\mathrm{c})$ and (d)] rates. The direction of the current was the same in both cases and it is marked by $I$ on the image. In (c), void formation in the right wire can be observed, which is the source of the material that is deposited at the junction.

results of EM strongly depend on the chosen rate. At a low rate $\left(\dot{G}_{T}=8 \mu \mathrm{S} / \mathrm{s}\right)$, shown in Fig. 5(b), the EM has removed the material from the narrowest point of the structure, as is expected. The observed contrast is due to PMMA leftovers used during fabrication of the nanodevices. ${ }^{28}$ The final conductance of this device was zero and clearly indicated the formation of a large gap. In this case, no care was taken to gently electromigrate once the junction size is reduced to an atomic size. Indeed, our focus was in the speed dependence of the electromigration process, rather than fabricating nanogaps. Nonetheless, in case the fabrication of controlled nanogaps is desired, our software has proven to be performant. At a much higher EM rate $\left(\dot{G}_{T}=60 \mu \mathrm{S} / \mathrm{s}\right)$, Figs. 5(c) and 5(d), a totally different result is obtained, i.e., the material is deposited at the narrowest region. The reproducibility of the effects was demonstrated conducting a few more experiments with high and low rates on the same type of structures with a similar output. It is important to notice that in the case of high EM rate the weakest region is not thinning but becomes even thicker. The obvious source of that agglomeration is the incoming material from the wire being electromigrated [Fig. 5(c)].

A requirement for void formation is the presence of a spatial gradient in the material transport. Since the material transport, given by Eq. (1), depends on the current density and temperature, both quantities could play an import role. At low electromigration rates, this gradient is provided by the change in current density while passing through the junction and a void is created at the constriction. However, considering the position of void formation at higher electromigration rates, i.e., higher EM powers and temperatures, this current density cannot be the sole quantity that determines the void formation. In this case, the presence of strong thermal gradients at a certain distance from the constriction could promote the observed void information. This effect should not be confused with thermomigration. Moreover, the larger amount of moving atoms, due to the increased EM power, cannot channel through the constriction and atoms get jammed. In order to confirm this speculation and fully understand the observed effect, more detailed modeling and/or experiments are needed.
The described effect limits not only the useful rate of EM but also the applicability of the ultra-fast EM control schemes like that reported by Kanamaru et al. ${ }^{12}$

\section{CONCLUSION}

In conclusion, we introduced a simple, and easy to implement, protocol to perform controlled electromigration, using different process variables. The software combines a PIDbased feedback loop to obtain the desired EM rate and a fast non-linear loop to prevent uncontrolled electromigration. The obtained data indicate that we are indeed able to electromigrate devices with a strong control of the EM speed and of the final conductance of the device. Furthermore, we have proven the strong dependence of an EM driven geometry change on the rate of electromigration. This underlines the importance of speed control for EM-based nanofabrication, which can be achieved by using the proposed algorithm.

\section{ACKNOWLEDGMENTS}

This work is supported by the KU Leuven Internal Funds Project No. CREA/14/011, the COST action NanoCoHybri (CA16218) and by the F.R.S.-FNRS (PDR T.0106.16).

${ }^{1}$ P. S. Ho and T. Kwok, Rep. Prog. Phys. 52, 301 (1989)

${ }^{2}$ J. Lloyd, Defect Diffus. Forum 143-147, 1661 (1997).

${ }^{3}$ D. Pierce and P. Brusius, Microelectron. Reliab. 37, 1053 (1997)

${ }^{4}$ A. Scorzoni, B. Neri, C. Caprile, and F. Fantini, Mater. Sci. Rep. 7, 143 (1991).

${ }^{5}$ R. Hoffmann-Vogel, Appl. Phys. Rev. 4, 031302 (2017).

${ }^{6}$ D. R. Strachan, D. E. Smith, D. E. Johnston, T. H. Park, M. J. Therien, D. A. Bonnell, and A. T. Johnson, Appl. Phys. Lett. 86, 043109 (2005).

${ }^{7}$ H. Park, J. Park, A. Lim, E. Anderson, A. Alivisatos, and P. McEuen, Nature 407, 57 (2000).

${ }^{8}$ W. Harneit, C. Boehme, S. Schaefer, K. Huebener, K. Fostiropoulos, and K. Lips, Phys. Rev. Lett. 98, 216601 (2007).

${ }^{9}$ X. D. A. Baumans, D. Cerbu, O.-A. Adami, V. S. Zharinov, N. Verellen, G. Papari, J. E. Scheerder, G. Zhang, V. V. Moshchalkov, A. V. Silhanek, and J. Van de Vondel, Nat. Commun. 7, 10560 (2016).

${ }^{10}$ Z. M. Wu, M. Steinacher, R. Huber, M. Calame, S. J. van der Molen, and C. Schönenberger, Appl. Phys. Lett. 91, 053118 (2007).

${ }^{11}$ J. M. Campbell and R. G. Knobel, Appl. Phys. Lett. 102, 023105 (2013).

${ }^{12}$ Y. Kanamaru, M. Ando, and J.-i. Shirakashi, J. Vac. Sci. Technol., B: Nanotechnol. Microelectron.: Mater., Process., Meas., Phenom. 33, 02B106 (2015).

${ }^{13}$ J. Black, Proc. IEEE 57, 1587 (1969).

${ }^{14}$ W. Jeong, K. Kim, Y. Kim, W. Lee, and P. Reddy, Sci. Rep. 4, 4975 (2014).

${ }^{15}$ H. B. Heersche, G. Lientschnig, K. O'Neill, H. S. J. Van Der Zant, and H. W. Zandbergen, Appl. Phys. Lett. 91, 072107 (2007).

${ }^{16}$ S. Talukder, A. Ghosh, and R. Pratap, J. ISSS 1, 16-22 (2012).

${ }^{17}$ A. A. Houck, J. Labaziewicz, E. K. Chan, J. A. Folk, and I. L. Chuang, Nano Lett. 5, 1685 (2005).

${ }^{18}$ G. Esen and M. S. Fuhrer, Appl. Phys. Lett. 87, 263101 (2005).

${ }^{19}$ O. Elíasson, G. Vasile, S. Ægir Jónsson, G. I. Gudjonsson, M. Arikan, and S. Ingvarsson, Rev. Sci. Instrum. 85, 114709 (2014).

${ }^{20}$ G. Kästle, H.-G. Boyen, A. Schröder, A. Plettl, and P. Ziemann, Phys. Rev. B 70, 165414 (2004).

${ }^{21}$ J. H. Lienhard, A Heat Transfer Textbook, 4th ed. (Dover Publications, 2011).

${ }^{22}$ C. J. M. Lasance, Heat Transfer Eng. 29, 149 (2008).

${ }^{23}$ H. S. J. Van Der Zant, E. A. Osorio, M. Poot, and K. O’Neill, Phys. Status Solidi B 243, 3408 (2006).

${ }^{24}$ K. J. Astrom and R. M. Murray, Feedback Systems: An Introduction for Scientists and Engineers (Princeton University Press, 2008). 
${ }^{25}$ X. Baumans, V. Zharinov, E. Raymenants, S. Alvarez, J. Scheerder, J. Brisbois, D. Massarotti, R. Caruso, F. Tafuri, E. Janssens et al., Sci. Rep. 7, 44569 (2017).

${ }^{26}$ X. Baumans, J. Lombardo, J. Brisbois, G. Shaw, V. Zharinov, G. He, H. Yu, J. Yuan, B. Zhu, K. Jin et al., Small 13, 1700384 (2017).

${ }^{27}$ S. P. Gurunarayanan, N. Verellen, V. S. Zharinov, F. J. Shirley, V. V. Moshchalkov, M. Heyns, J. Van de Vondel, I. P. Radu, and P. Van Dorpe, Nano Lett. 17, 7433 (2017).
${ }^{28}$ D. S. Macintyre, O. Ignatova, S. Thoms, and I. G. Thayne, J. Vac. Sci. Technol., B: Microelectron. Nanometer Struct.-Process., Meas., Phenom. 27, 2597 (2009).

${ }^{29}$ T. Taychatanapat, K. I. Bolotin, F. Kuemmeth, and D. C. Ralph, Nano Lett. 7, 652 (2007)

${ }^{30}$ J. Lombardo, Ž. L. Jelić, X. D. A. Baumans, J. E. Scheerder, J. P. Nacenta, V. Moshchalkov, J. Van de Vondel, R. B. G. Kramer, M. V. Milosevic, and A. V Silhanek, Nanoscale 10, 1987 (2018). 